\title{
Collective-Intelligence and Decision-Making
}

\author{
Paulo Trigo ${ }^{1}$ and Helder Coelho ${ }^{2}$ \\ 1 ISEL - Instituto Superior de Engenharia de Lisboa, Portugal \\ DEETC - Dep. de Eng. de Electr. e Telec. e de Computadores \\ GuIAA (DEETC), LabMAg (FCUL) \\ ptrigo@deetc.isel.ipl.pt \\ 2 FCUL - Faculdade de Ciências da Univ. de Lisboa, Portugal \\ DI - Departamento de Informática \\ LabMAg (FCUL) \\ hcoelho@di.fc.ul.pt
}

\begin{abstract}
Decision-making, while performed by humans, is also expected to be found in most (artificial) intelligent systems. Usually, the cognitive research assumption is that the individual is the correct unit for the analysis of (human) intelligence. Yet, the multi-agent assumption is that of a society of interacting individuals (agency) that collectively supersedes individual capabilities. Therefore, the entire society of agents is, itself, an additional locus for the analysis of this collective-intelligence. In this paper we propose models that explore the agent-agency mutual influence from the decision-making perspective. We outline three case study scenarios used during the model's experimental evaluation: i) large-scale disasters, ii) electricity markets, and iii) Web-empowered knowledge and social connectivity. The scenario-driven evaluations are being used to provide the alignment weights for the effort along our research lines.
\end{abstract}

\section{Introduction}

"What do you think about our course in this institution? - the younger student asks two older students while looking at a professor that roves nearby." This daily, face-to-face, scene enfolds individuals in two major ways: i) throughout knowledge sharing, and ii) by refining (clarifying) their social network connectivity. Now, imagine that same student exploring the Internet, e.g., via search engines, electronic encyclopedias or Web logs (blogs), looking for answers and also providing his own opinion on that same question. In both scenes (face-to-face and Internet) each individual's decision-making influences others while also being influenced by them. The resulting overall knowledge and group reasoning capability supersedes individuals and frames a collective-intelligence.

There are several perspectives on the collective-intelligence concept. A behavioral approach considers collective intelligence as "consisting of a large number of quasi-independent, stochastic agents, interacting locally both among themselves and with an active environment, in the absence of hierarchical organization, and yet capable of adaptive behavior" [21]. The organizational perspective 
of collective-intelligence is that of "groups of individuals doing things collectively that seem intelligent" [12].

The different collective-intelligence perspectives contribute to formulate a basic research question: "How can people and computers be connected so that collectively - they act more intelligently than any individuals, groups, or computers have ever done before?" [14]. This question brings humans and computers as partners mutually influencing each other; it is not just the computer that exhibits intelligent capabilities but the blending of computers with humans that aims to exceed each other's capabilities. The collective-intelligence has always been around but the modern "computer partnership" exposes new insights on such "blending" (of computers and humans) thus being the major novelty and driving force for the collective-intelligence research field.

In this paper we describe our research proposals on the multi-agent decision-making field, mainly focused on domains that exhibit uncertain causal effects. Section 2 describes the essentials of our proposal to decision-making with multiple simultaneous goals from an institutional agent's perspective. In section 3 we extend the previous scope and formulate a model that accounts for the separation of concerns between collective and individual motivations, thus framing a 2 -strata decision model, collective 'versus' individual $(\mathrm{CvI})$ where the definition of inter-stratum relations enables each agent to choose at which stratum each decision should be taken. The section 4 simplifies the CvI model and exploits it in the electricity market domain with the multi-agent simulator TEMMAS (The Electricity Market Multi-agent Simulator) and its market agents. The last proposal is presented in section 5 with our preliminary work to exploit the crosscutting between the agent-based system modeling and the Web-based methodologies given the emphasis on the emerging ideas (and tools) that populate the Semantic Web (SW) field. Institutional agents can be designed to take advantage of the collective-knowledge-representation perspective of the Internet space and to foster the early adoption, by the institutions, of the SW concepts and technologies, thus expanding the organizations' collective-intelligence.

\section{Multiple simultaneous goals and uncertain causality}

The mitigation of a large-scale disaster, caused either by a natural or a technological phenomenon (e.g., an earthquake or a terrorist incident), gives rise to multiple simultaneous goals that demand the immediate response of a finite set of specialized agents. In order to act rationally the agent must evaluate multiple and simultaneous perceived damages, account for the chance of mitigating each damage and establish a preferences relation among goals. Although rational behavior stands on the capability to establish preferences among simultaneous goals, current belief-desire-intention (BDI) mental-state approaches [18, 31] (widely used as reasoning agents' foundations) do not provide a theoretical or architectural framework for predicting how goals interact and how an agent decides which goals to pursue. When faced with multiple simultaneous goals, the BDI's intention selection process (decision) follows a heuristic approach, usually 
coded by a human designer [16]. Additionally, BDI models find it difficult to deal with uncertainty, hence hybrid models have been proposed combining BDI and Markov decision process (MDP) approaches [20, 23]; however, hybrid models usually assume that the goal has already been chosen and tackle the stochastic planning problem (in order to achieve the chosen goal).

In this work we take the decision-theoretic notion of rationality to estimate the importance of goals and to establish a preferences relation among multiple goals. We propose a preferences model that allows agent developers to design the relationships between perceived (certain) and uncertain aspects of the world in an easy and intuitive manner. The design is founded on the influence diagram [10] (ID) framework that combines uncertain beliefs and the expected gain of decisions. The proposal's practical usefulness is experimentally explored in a fire fighting scenario in the RoboCupRescue [11] domain. The decision model incorporates general fire fighting principles in a way that considerably simplifies the specification of a preferences relation among goals. Despite such simplification, the attained results are consistent with the initial fire fighting principles. For the comprehensive description of the proposal we refer to [24].

\subsection{The preferences model and the causal effect pattern}

The premisse of the preferences model is that the relation among simultaneous goals follows from the expected utility of the available decisions. The expected utility of any available decision combines: i) the observed state value, and ii) the likelihood of success of that decision. Given a set of available decisions, $\mathcal{D}$, a set of states, $\mathcal{S}$, an utility function, $u: \mathcal{S} \rightarrow \mathbb{R}$, and the probability, $P(s \mid d)$, to achieve state $s \in \mathcal{S}$ after decision $d \in \mathcal{D}$, the expected utility, eu $: \mathcal{D} \rightarrow \mathbb{R}$, of decision-making is described by, $e u(D=d)=\sum_{s \in \mathcal{S}} P(s \mid D=d) u(s)$, where $D$ is a variable that holds an available decision.

Given any goal there are always two available decisions: i) pursue the goal, or ii) ignore the goal. Thus, $\mathcal{D}=\{$ yes, no $\}$, is such that $D_{g}=$ yes and $D_{g}=$ no represent, respectively, the decision to pursue or to ignore the goal $g \in \mathcal{G}$. The utility of a goal, $g$, measures the importance, assigned by the agent, to the goal $g$. The "importance" is a criterion related to a valuation in terms of benefits and costs an agent has of a mental state situation [5]. The goal achievement payoff is estimated by the difference between the expected utility on pursuing and ignoring that goal. Thus, the goal utility function, $u_{\mathcal{G}}$, for each $g \in \mathcal{G}$, is defined by, $u_{\mathcal{G}}(g)=e u\left(D_{g}=\right.$ yes $)-e u\left(D_{g}=\right.$ no $)$. The agent prefers goal $g_{1}$ over $g_{2}$ (i.e., $g_{1} \succ g_{2}$ ) if $g_{1}$ offers higher payoff and when even, prefers the goal that, when achieved, gives higher expected advantage (i.e., higher $e u\left(D_{g}=\right.$ yes $)$ ). In sight of equality the agent is indifferent between goals (i.e., $g_{1} \sim g_{2}$ ), thus taking, for instance, an exploratory decision.

The causal effect pattern. The causal effects (consequences) of each decision are unknown, therefore our aim is to choose the decision alternative (goal) that minimizes the eventual disadvantageous consequences of such decision. The influence diagram (ID) framework combines uncertain beliefs to compute the expected 
utility of decisions, thus rationality is a matter of choosing the alternative that leads to the highest expected utility, given the evidence of available information. The ID extends the Bayesian's network chance nodes with two additional nodes: decisions and utilities, and two additional arcs: influences and informational. We propose a set of (nine) guidelines, to build an ID pattern to describe the multiple and simultaneous goals decision problem. Figure 1 illustrates the pattern using the regular ID symbols; circle is a chance node, rectangle is a decision node and the lozenge is an utility node.

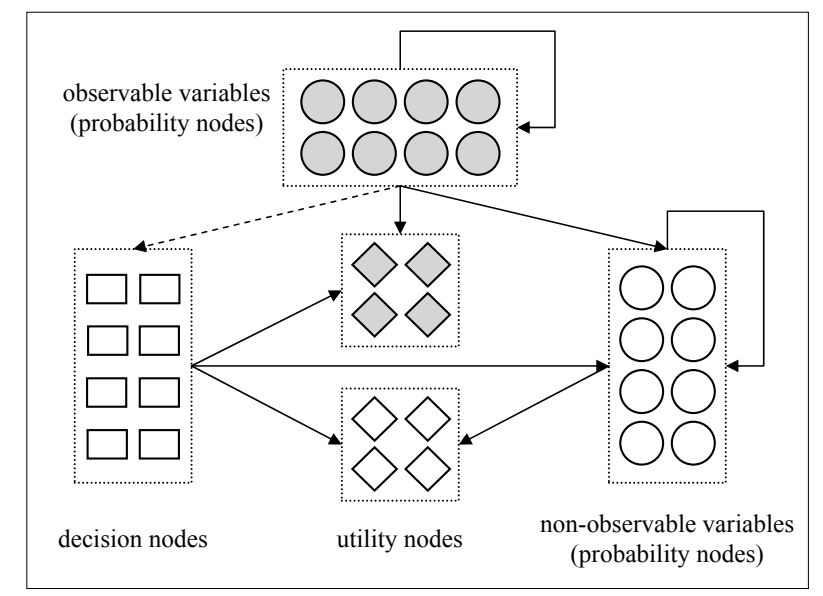

Fig. 1. The influence diagram (ID) pattern (the sets are represented by dotted rectangles; the gray elements refer to observable information; the dotted arcs are informational arcs and the remaining are conditional arcs).

The gray filling (cf. figure 1) has a special meaning: i) the gray chance node indicates information availability, i.e., an observable variable, and ii) the gray utility node indicates a dependency from a gray chance node, i.e., the utility of some observable variables. The sets of nodes with similar characteristics are aggregated by a dotted rectangle. The arcs connect sets of nodes (instead of individual nodes), therefore attaining an ID pattern, i.e., a template from which to build several different instances with the same overall structure; we next describe the ID pattern usage.

\subsection{The experimental scenario (multiple simultaneous goals)}

We used the RoboCupRescue environment to devised a disaster scenario that evolves at the Nagata ward in Kobe, Japan. Two buildings, $B_{1}$ and $B_{2}$, not far from each other (about 90 meters) catch a fire. The $B_{1}$ is relatively small and is located near Kobe's harbor, in a low density neighborhood. The $B_{2}$ is of medium size and it is highly surrounded by other buildings. As time passes, the 
fires' intensity increase so a close neighbor is also liable to catch a fire. Figure 2 shows the disaster scenario; each opaque rectangle is a building and a small circle is positioned over $B_{1}$ and $B_{2}$. The two larger filmy squares define the neighborhood border of $B_{1}$ and $B_{2}$ within a $d$ distance (in meters).

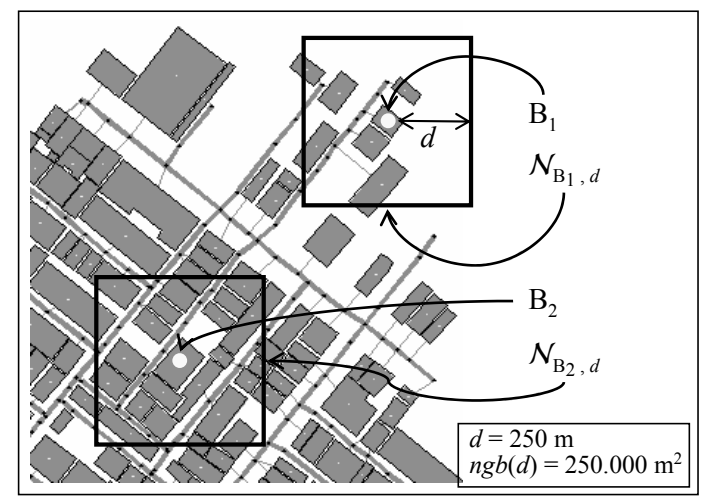

Fig. 2. Fire scenario in the buildings labeled $B_{1}$ and $B_{2}$ (the set of buildings that are contained within each building's neighborhood, $n g b(d)$, is represented by $\left.\mathcal{N}_{B_{i}, d}\right)$.

To simplify we assume that: i) buildings use identical construction materials, ii) buildings are residential (neither offices nor industries inside the buildings), and iii) there are no civilians, caught by fires, inside the buildings. In order to apply the ID pattern (cf. figure 1) to the illustrative scenario (cf. figure 2) we formulated, for each building, the observable and non-observable variables along with the utility nodes which followed three general fire attack strategies that, although intuitive, were acquired after the RoboCupRescue experimentation.

Results and prospects after this work. Practical experiences indicate that the ID pattern considerably simplifies the specification of a decision model (in the RoboCupRescue domain) and enabled to established a preferences order among goals that is consistent with the initial, domain expert, very general strategies. This work represents the ongoing steps in a line of research that aims to develop decision-making agents that inhabit complex environments (e.g., the RoboCupRescue). Agter this work we applied the preferences model to the problem of coordinating teamwork (re)formation [23] from a centralized perspective.

\section{Decisions with collective and individual motivations}

The agents that cooperate to mitigate the effects of a large-scale disaster take decisions that follow two large behavioral classes: the individual (ground) activity and the collective (institutional) coordination of such activity. However, despite the intuition on a 2-strata (collective and individual) decision process, research 
on multi-agent coordination often proposes a single model that amalgamates those strata and searches for optimality within that model.

In this work we propose the multi-agent collective 'versus' individual (CvI) decision model, which is neither purely collective nor purely individual, is founded on the semi-Markov decision process (SMDP) framework and is designed to explore the explicit separation of concerns between both (collective and individual) decision strata while aiming to conciliate their reciprocal influence. The comprehensive description of the CvI model refers to [28].

\subsection{The collective 'versus' individual (CvI) decision model}

The premise of the CvI decision model is that the individual choice coexists with the collective choice and that coordinated behavior happens (is learned) from the prolonged relation (in time) of the choices exercised at both of those strata (individual and collective). Additionally, coordination is exercised on high level cooperation tasks, represented within an hierarchical task organization. The tasks' hierarchy is founded on the framework of Options [22], which extends the MDP theory to include temporally abstract actions, i.e., variable time duration tasks, whose execution resorts to a subset of primitive actions.

The CvI collective and individual strata. The individual stratum is simply a set of agents, $\Upsilon$, each agent, $j \in \Upsilon$, having its particular capabilities described as an hierarchy of options. The CvI model admits agent heterogeneity (diverse option hierarchies), as long as all hierarchies have the same number of levels (depth), i.e., a similar temporal abstraction is used to design all hierarchies.

The collective stratum consists of a single agent (e.g. an institutional agent) that represents the whole set of individual stratum agents. The collective stratum agent cannot act on its own; its actions must be materialized through the individual stratum agents. The purpose of the collective stratum is to coordinate the individual stratum. Formally, at the collective stratum, each action is defined as a collective option, $o_{\vec{o}}=\left\langle\mathcal{I}_{\vec{o}}, \pi_{\vec{o}}, \beta_{\vec{o}}\right\rangle$, where $\vec{o}=\left\langle o^{1}, \ldots, o^{|\Upsilon|}\right\rangle$ represents the simultaneous execution of option $o^{j} \equiv\left\langle\mathcal{I}^{j}, \pi^{j}, \beta^{j}\right\rangle$ by each agent $j \in \Upsilon$. The set of agents, $\Upsilon$, defines an option space, $\overrightarrow{\mathcal{O}} \subseteq \mathcal{O}^{1} \times \ldots \times \mathcal{O}^{|\Upsilon|}$, where $\mathcal{O}^{j}$ is the set of agent $j$ options and each $o_{\vec{o}} \in \overrightarrow{\mathcal{O}}$ is a collective option. The $\overrightarrow{\mathcal{O}}$ decomposes into $\overrightarrow{\mathcal{O}}_{d}$ disjoint subsets, each containing only the collective options available at the, $d$, hierarchical level, where $0<d \leq D-1$ and level-0 is the hierarchy root, at which there are no options to choose from, and level- $D$ is the hierarchy depth. A level $d$ policy, $\pi_{d}$, is implicitly defined by the SMDP $\mathcal{M}_{d}$ with state set $\mathcal{S}$ and action set $\overrightarrow{\mathcal{O}}_{d}$. The $\mathcal{M}_{d}$ solution is the optimal way to choose the level $d$ individual policies which, in the long run, gathers the highest collective reward.

The CvI structure and dynamics. Figure 3 illustrates the CvI structure where the individual stratum (each agent $t^{j}$ ) is a 3-level task hierarchy and thus the collective stratum is a 2-level hierarchy; at each level, the set of diamond ended arcs, links the collective option to each of its individual policies. 


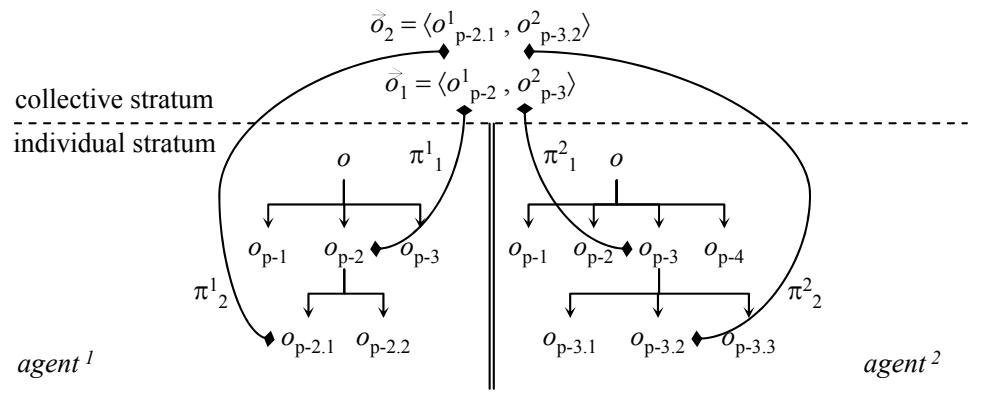

Fig. 3. The structure of the CvI decision model and links between strata (superscript $j$ refers to agent ${ }^{j}$; subscripts $k$ and $p$ - $k$ refer to $k$ hierarchical level and $k$ tree path).

Figure 4 illustrates the CvI dynamics; at each decision epoch, agent ${ }^{j}$ gets the partial perception, $\omega^{j}$, and decide-who-decides $(d-w$ - $d)$, i.e., the agent ${ }^{j}$ either: i) chooses an option $o^{j} \in \mathcal{O}^{j}$, or ii) requests, the collective stratum, for a decision. The collective stratum always replies with an option, $o^{j}$, decision. The $d-w$ - $d$ process represents the importance that an agent credits to each stratum motivation, which is materialized as the ratio between, the maximum expected benefit in choosing a collective and an individual decision. The expected benefit is given, at each hierarchical level- $d$, by the value functions of the corresponding SMDP $\mathcal{M}_{d}$. A threshold, $\kappa \in[0,1]$, supports the focus-grade between collective and individual strata. Such regulatory mechanism enables the (human) designer to specify diverse social attitudes: ranging from common-good $(\kappa=0)$ to self-interested $(\kappa=1)$ motivated agents.

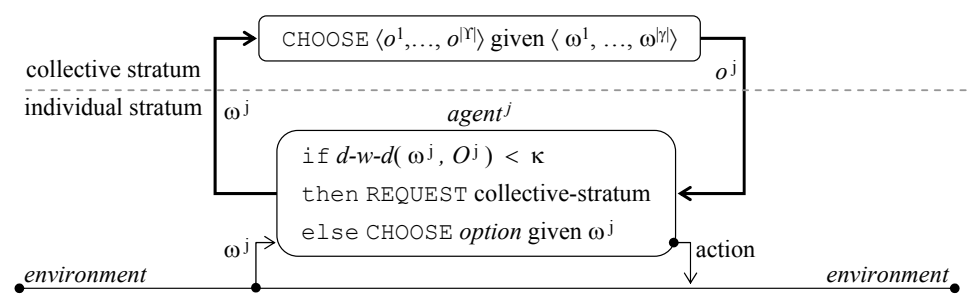

Fig. 4. The CvI decide-who-decides $(d-w$ - $d)$ process ( $o \equiv$ option; $\mathcal{O} \equiv$ option set; $\omega \equiv$ partial observation; superscript $j$ refers to agent $t^{j}$ )

\subsection{The experimental scenario (ambulances and injured civilians)}

We implemented the CvI decision model and tested it in a multi-agent ambulance environment: "a maze-like grid inhabited by ambulances (or taxis), injured civilians (or passengers) and hospitals (or sites)". The problem was originally formulated by Dietterich [6] as a single-agent problem to explore the properties of 
a hierarchical reinforcement learning method. We extended the original problem to the multi-agent environment as follows: "the environment is inhabited by multiple taxis (the agents), multiple passengers and a set of sites; a site may be the origin of several passengers, each one with its own destination; each taxi is able to pick up and drop down several passengers; each taxi may simultaneously transport several passengers" [28]. The environment is individually partially observable as each agent does not perceive the other agents' locations; also, the environment is collectively observable as the combination of all individual observations determines a sole world state. The goal of the individual stratum is to learn how to execute tasks (e.g. how to navigate to a site and when to pick up a passenger). The goal of the collective stratum is to learn to coordinate those individual tasks as to minimize the resources (time) to satisfy the passengers' needs. The learning of the policy at the collective stratum occurs simultaneously with the learning of each agent's policy at the individual stratum.

Results and prospects after this work. The formulation of the CvI multilevel hierarchical decision model and the definition of an inter-strata regulatory mechanism enable us to show experimentally how to explore the individual policy space in order to decrease the complexity of learning a coordination policy in a partially observable setting. The 2 -strata approach augments the design flexibility in two ways: i) makes it possible to specify individual task hierarchies that are not necessarily equal, therefore allowing for agents' heterogeneity, and ii) enables to configure different architectures (e.g. centralized or decentralized) depending on the information exchange between collective and individual layers.

Our contribution was extended with the formulation of the CvI-JI hybrid model [27]. We have identified a series of relations between the CvI decision-theoretic approach and the joint-intentions (JI) [4] mental-state based reasoning. The CvI was extended by exploring the algorithmic aspects of the CvI-JI integration. Such integration represents our novel contribution to a multi-agent hybrid decision model within a reinforcement learning framework.

\section{Decision-making for electricity markets}

The start-up of nation-wide electric markets, along with its recent expansion to intercountry markets, aims at providing a competitive electricity service to consumers. This newly deregulated electricity market organization calls for an increasing (human) decision-making responsibility in order to settle the energy assets' trading strategies. The growing number of interactions among market participants and their mutual-influencing are usually described by game theoretic approaches which are based on the determination of equilibrium points against which to compare the actual market performance [3, 7]. However, those approaches find it difficult to incorporate the ability of market participants to repeatedly probe markets and adapt their strategies. As an alternative to the equilibrium approaches, the multi-agent based simulation (MABS) comes forth as being particulary well fitted to analyze dynamic and adaptive systems with complex interactions among constituents $[19,9]$. 
In this work we propose a MABS to capture the behavior of the electricity market and to build TEMMAS (The Electricity Market Multi-agent Simulator), which incorporates the operation of multiple generator company ( $\mathrm{GenCo}$ ) operators, each with distinct power generating units (GenUnit), and a market operator $(\mathrm{Pool})$ which computes the hourly market price (driven by the electricity demand). We used TEMMAS to construct an agency for the Iberian (Portugal and Spain) Electricity Market (MIBEL - "Mercado Ibérico de Electricidade"). The agency follows the market organizational structure and each agent acts (bid, buy, sell) according to its role in the market. The simulation explores the relation between the production capacity and the search for competitive biding strategies. Results show a coherent market behavior as price reductions from competition favour consumers and harm producers that do not adapt their electricity bids. The comprehensive description of TEMMAS refers to [25, 29].

\subsection{TEMMAS agency design}

Within the current design model of TEMMAS the electricity asset is traded through a spot market (no bilateral agreements), which is operated via a Pool institutional power entity. Each generator company, GenCo, submits (to Pool) how much energy, each of its generating unit, GenUnit ${ }_{G e n C o}$, is willing to produce and at what price. The bidding procedure conforms to the so-called "block bids" approach [15], where a block represents a quantity of energy being bided for a certain price; also, GenCos are not allowed to bid higher than a predefined price ceiling. Thus, the market supply essential measurable aspects are the energy price, quantity and marginal production cost. The consumer side of the market is mainly described by the quantity of demanded energy; we assume that there is no price elasticity of demand (i.e., no demand-side market bidding). The Pool is a reactive agent that always applies the same predefined auction rules in order to determine the market price and hence the block bids that clear the market. Each $\mathcal{E}_{\text {GenUnit } t_{\text {GenCo }}}$ represents the $G e n C o$ 's set of available resources. The $\mathcal{E}_{\text {GenCo }}$ contains the decision-making agents.

Decision-making and adaptation. Each generator company defines the bidding strategy for each of its generating units. We designed two types of strategies: a) the basic-adjustment, that chooses among a set of basic rigid options, and b) the heuristic-adjustment, that selects and follows a predefined well-known heuristic. The strategies correspond to the GenCo agent's primary actions. The GenCo has a set, $\mathcal{E}_{\text {GenUnit }}{ }_{G e n C o}$, of generating units and, at each decision-epoch, it decides the strategy to apply to each generating unit, thus choosing a vector of strategies, $\overrightarrow{s t t g}$, where the $i^{\text {th }}$ vector's component refers to the GenUnit ${ }_{G e n C o}^{i}$ generating unit. The GenCo's perceived market share, mShare, is used to characterize the agent internal memory so its state space is given by mShare $\in$ [0..100]. Each GenCo is a MDP decision-making agent such that the decision process period represents a daily market. At each decision-epoch each agent computes its daily profit (that is regarded as an internal reward function) and the Pool agent receives all the GenCos's block bids for the 24 daily hours and settles the hourly 
market price by matching offers in a classic supply and demand equilibrium price (we assume a hourly constant demand).

TEMMAS architecture and construction. Figure 5 shows TEMMAS agents along with the major inter-agent communication paths; at the top there is an user interface to specify each of the resources' and agents' configurable parameters.

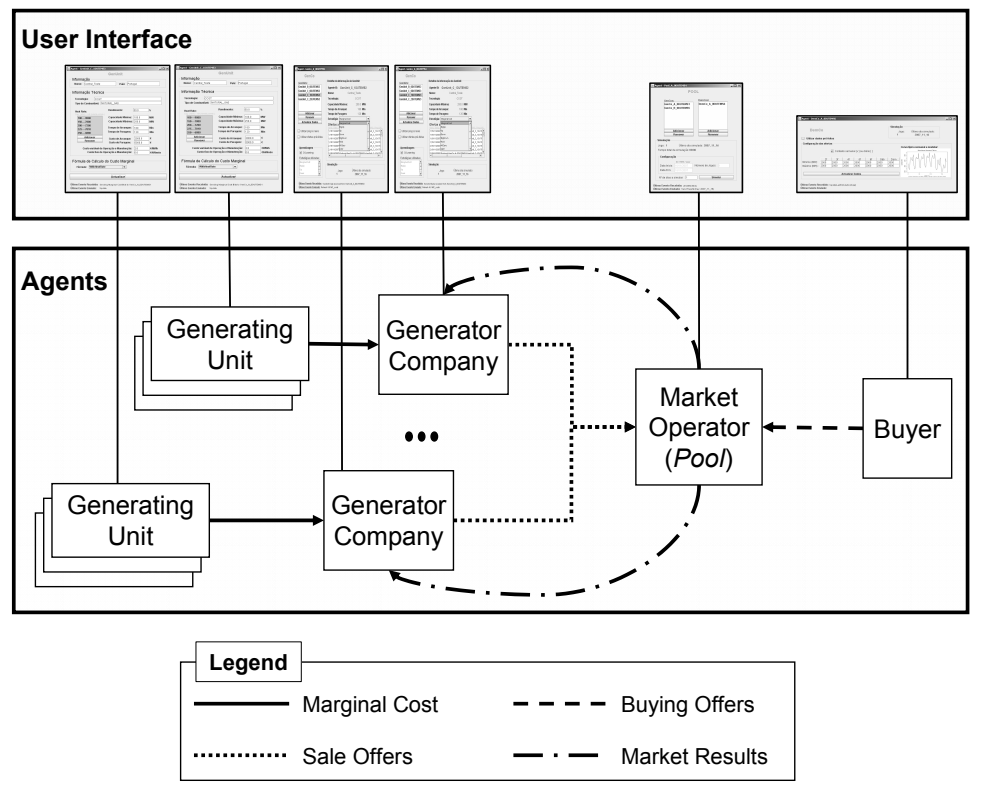

Fig. 5. The TEMMAS architecture and the configurable parameters.

The implementation of the TEMMAS architecture followed the INGENIAS [8] methodology and used its supporting development platform.

\subsection{The experimental scenario (Iberian electricity market)}

We used TEMMAS to build a specific electric market simulation model taken from the Iberian Electricity Market (MIBEL - "Mercado Ibérico de Electricidade") with Portuguese (e.g., EDP - "Electricidade de Portugal", "Turbogás", "Tejo Energia") and Spanish (e.g., "Endesa", "Iberdrola", "Union Fenosa", "Hidro Cantábrico", "Viesgo", "Bas Natural", "Elcogás") generator companies. Regarding the total electricity capacity installed the Iberian market is composed of a major player (Spain) and a minor player (Portugal). Figure 6 uses INGENIAS notation to depict the hierarchical structure of the electricity market. We considered three types of generating units: i) coal plant, $\mathrm{CO}$, to provide the base load demand, ii) combined cycle plant, CC, to cover intermediate load, and iii) gas turbine, GT, to cover peaking loads. 


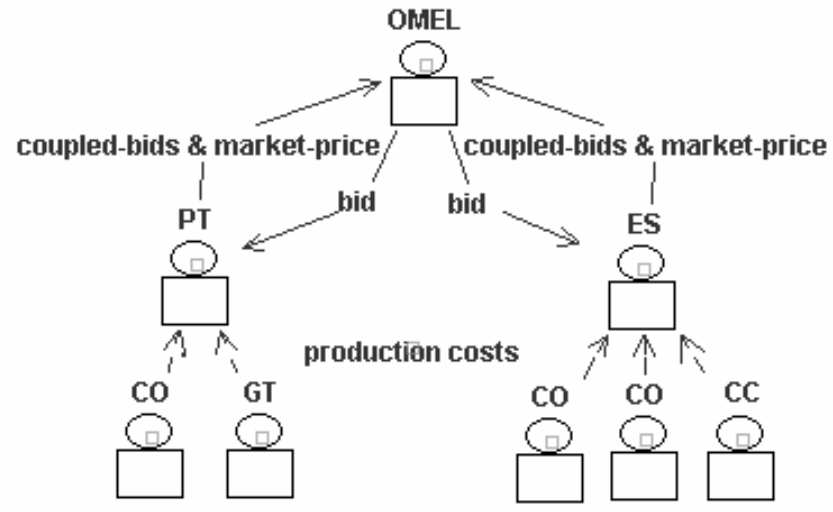

Fig. 6. An illustrative TEMMAS formulation (using INGENIAS notation); PT and ES denote, respectively, Portugal and Spain; OMEL represents the Iberian market oprator.

The simulation shows how a one-company market will find its way to sell all its electricity at the price of its most expensive energy (e.g., sell the cheapest $\mathrm{CO}$ at the price of the expensive CC and GT). The results for the two-company market show that a big company, capable of supplying the whole demand, with a strategy to always settle the market price will probably loose some of its market share for a smaller but aggressive cutting-price competitor. This may also suggest that smaller companies could explore alternative ways to increase its market share, such as attractive bilateral contracts with final consumers.

Results and prospects after this work. TEMMAS supports multiple generator companies, multiple power plant with different technologies and distinct ways of computing marginal costs. The user is given the capability to configure the decision-making agents (specify the bidding strategies or choose from a set of predefined); also decision-making agents may be configured as learning or nonlearning agents. The system is being specified (from the beginning) as a multi-agent environment [25], modeled with INGENIAS, implemented with JADE [2] (using "INGENIAS to JADE" transformations and additional Java coding) and with R-Project [30] for the statistical computations and graphics' generation. Although TEMMAS is currently in a preliminary stage its architectural and design decisions are strongly founded in the MABS field and the initial results are an incentive to further extend the already implemented system. We intend to extend TEMMAS taking into account the particularities of the MIBEL market (e.g., the network congestion and its relation with the market-splitting procedure).

\section{Agent inferencing meets the Semantic Web}

Agent inferencing over Semantic Web (SW) descriptions gives organizations the opportunity to use the Internet as a "huge" collective-knowledge-representation 
platform. The meaning of this is clear: "there is an extended spectrum of applications and technologies for storing, searching and retrieving, data, information and knowledge"; also, management is given the means to achieve a more interactive, collaborative and decentralized decision-making profile. The progress towards the SW appears in the form of the standardization of languages and in the increasing maturity of related models and tools. The SPARQL (SPARQL Protocol and RDF Query Language) enables to extract the RDF (Resource Description Language) explicitly stored information while the SW rules (or semantic constraints) claim for ways of extracting the implicitly represented information.

In this work we propose a simple way of combining the SPARQL expressiveness with the RDF-triple homogeneous way of representing information in order to transform implicit knowledge into explicitly stored information. Our work takes a practical stance and assumes that the currently available SPARQL specifications $[1,17]$ do not support RDFS entailment. Therefore, we combine the SPARQL and RDF specifications [13] (also resorting to RDFS semantic constraints) to build an inferencing scenario driven by the ongoing SW appeal. The comprehensive description of this proposal refers to [26].

\subsection{The inference process}

The SPARQL language queries the information explicitly represented within an RDF graph. The implicit RDF triples are the ones that may be inferred from the rules that characterize each domain interpretation. The inference process considers the following typical representative of the rule format: $(\alpha \Rightarrow \beta)$ where $\alpha$ is the rule antecedent formulated as a conjunction of several RDF triples and $\beta$ is the rule consequent formulated as a single RDF triple. The inference process expands a graph $\mathcal{G}$ into a graph $\mathcal{G}^{\prime}$ which explicitly includes the triples that, given rule $(\alpha \Rightarrow \beta)$, only existed implicitly in $\mathcal{G}$.

\subsection{The experimental scenario (Fire-Brigade decision-making)}

The real-world institutions related with fire-brigade domain can clearly take advantage of SW early adoption. Those institutions rely on diverse sources of information thus depending on (automated) mechanisms for concepts' interpretation, classification and integration. For example, the Portuguese SNBPC (Serviço Nacional de Bombeiros e Protecção Civil - "National Fire Service and Civil Defense") must interpret (and integrate) the information provided by the set of fire-brigade command centers in order to plan and coordinate the huge amount of (annual) florestal fires; automated knowledge-handling would certainly be a helper "to follow the ambition on reducing" the (typical) Portuguese post-summer annual landscape of grievously huge burnt areas.

The fire-brigade domain contains several fire-brigade centers each being responsible for a certain physical region that holds a set of smaller places (e.g., house blocks within a town). The fires are classified according to their priority and several strategies have been devised for scenarios with several simultaneous fires [24] while the decision-making process follows the hierarchical relations 
among agents [27]. Figure 7 shows the basic RDF to describe a fire-brigade domain. Each arc is a property and each oval shaped node is a resource, the node $\mathrm{b}$ represents an anonymous resource (i.e., a blank node), the rectangle shaped node $\mathrm{v}$ represents a literal and the qualifier $\mathrm{D}$ represents the domain namespace.

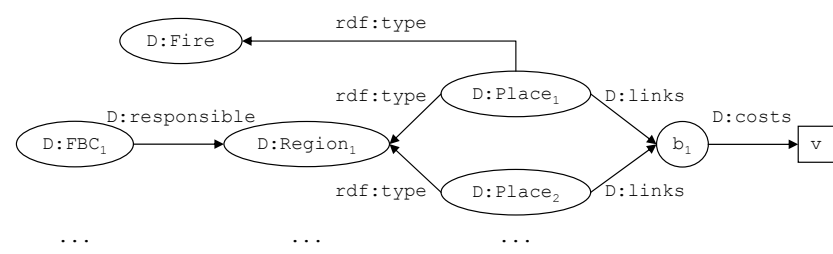

Fig. 7. The RDF basic graph structure of the fire-brigade domain.

The agent designer uses the RDF framework to describe the domain resources and properties and then he defines the semantic rules. When the agent receives a fire report the inference process is executed to determine the region of the fire and thus the fire-brigade center that has the responsibility of attacking the fire.

Results and prospects after this work. This is a preliminary work that is currently being developed in order to exploit the crosscutting between the agent-based system modeling and the Web-based methodologies given the emphasis on the emerging ideas (and tools) that populate the Semantic Web field. Institutional agents (e.g., fire-brigade) can be designed to take advantage of the collective-knowledge-representation perspective of the Internet space and to foster the early adoption, by the institutions, of the SW concepts and technologies.

\section{Conclusions}

This paper describes our research effort along the lines of individual and collective autonomous decision-making. We have designed and implemented agents that decide and act collectively in (simulated) large scale desastres, energy markets and in the semantic Web space. Our research effort have been strongly impelled by the question on "How to capture the dynamics of the agent-agency mutual influencing in decision-making processes?" The collective 'versus' individual (CvI) model is our most thorough proposal but humans are not regarded as full participants in the decision-making processes, i.e., the agency excludes humans (thus being purely virtual). The collective-intelligence perspective of "blending" humans and computers frees agents from the responsibility of substituting human processes. Both humans and computers regard the other as an appendix of itself. We intend to research new ways of augmenting our decision-making models with this "blending" modern way of looking at the collective-intelligence phenomenon. 


\section{References}

1. D. Beckett and J. Broekstra. SPARQL query results XML format. W3C Recommendation, Jan. 2008.

2. F. L. Bellifemine, G. Caire, and D. Greenwood. Developing Multi-Agent Systems with JADE. Wiley Series in Agent Technology. Wiley, April 2007.

3. C. Berry, B. Hobbs, W. Meroney, R. O'Neill, and W. S. Jr. Understanding how market power can arise in network competition: a game theoretic approach. Utilities Policy, 8(3):139-158, September 1999.

4. P. Cohen and H. Levesque. Teamwork. Noûs, Special Issue on Cognitive Science and Artificial Intelligence, 25(4):487-512, 1991.

5. M. Corrêa and H. Coelho. Collective mental states in extended mental states framework. In Proceedings of the IV International Conference on Collective Intentionality, Certosa di Pontignano, Siena, Italy, October 13-15 2004.

6. T. Dietterich. Hierarchical reinforcement learning with the MAXQ value function decomposition. Journal of Artificial Intelligence Research, 13:227-303, 2000.

7. S. Gabriel, J. Zhuang, and S. Kiet. A Nash-Cournot model for the north american natural gas market. In Proceedings of the 6th IAEE European Conference: Modelling in Energy Economics and Policy, 2-3 September 2004.

8. J. Gómez-Sanz, R. Fuentes-Fernández, J. Pavón, and I. García-Magariño. INGENIAS development kit: a visual multi-agent system development environment (BEST ACADEMIC DEMO OF AAMAS'08). In Proceedings of the Seventh AA$M A S$, pages 1675-1676, Estoril, Portugal, May 12-16 2008.

9. A. Helleboogh, G. Vizzari, A. Uhrmacher, and F. Michel. Modeling dynamic environments in multi-agent simulation. JAAMAS, 14(1):87-116, 2007.

10. R. Howard and J. Matheson. Influence diagrams. In Readings on the Principles and Applications of Decision Analysis, volume 2, pages 721-762. Strategic Decision Group, Menlo Park, CA, 1984.

11. H. Kitano and S. Tadokoro. RoboCup Rescue: A grand challenge for multi-agent systems. Artificial Intelligence Magazine, 22(1):39-52, 2001.

12. T. Malone, R. Laubacher, and C. Dellarocas. Harnessing crowds: Mapping the genome of collective intelligence. Working paper 2009-001, MIT Center for Collective Intelligence, Feb. 2009.

13. F. Manola and E. Miller. RDF primer. W3C Recommendation, Feb. 2004.

14. MIT. MIT Center for Collective Intelligence. http://cci.mit.edu/, 2009.

15. OMIP. Iberian Electricity Market Operator. online: 'www.omip.pt'.

16. A. Pokahr, L. Braubach, and W. Lamersdorf. A goal deliberation strategy for BDI agent systems. In Proceedings of the Third German Conference on Multi-Agent System Technologies (MATES-2005), pages 82-94. Springer, 2005.

17. E. Prud'hommeaux and A. Seaborne. SPARQL query language for RDF. W3C Recommendation, Jan. 2008.

18. A. Rao and M. Georgeff. BDI agents: From theory to practice. In Proceedings of the First International Conference on Multiagent Systems, pages 312-319, San Francisco, USA, 1995.

19. S. Schuster and N. Gilbert. Simulating online business models. In Proceedings of the 5th Workshop on Agent-Based Simulation (ABS-04), pages 55-61, May 3-5 2004 .

20. G. Simari and S. Parsons. On the relationship between MDPs and the BDI architecture. In Proceedings of the Fifth International Joint Conference on Autonomous Agents and Multiagent Systems (AAMAS-06), pages 1041-1048, Hakodate, Japan, May 8-12 2006. ACM Press. 
21. W. Sulis. Fundamental concepts of collective intelligence. Nonlinear Dynamics, Psychology, and Life Sciences, (1):35-53, Jan. 1997.

22. R. Sutton, D. Precup, and S. Singh. Between MDPs and semi-MDPs: A framework for temporal abstraction in reinforcement learning. Artificial Intelligence, 112(12):181-211, 1999.

23. P. Trigo and H. Coelho. Decision making with hybrid models: the case of collective and individual motivations. In Proceedings of the EPIA-07 International Conference (New Trends in Artificial Intelligence), pages 669-680, Guimarães, Portugal, 2007.

24. P. Trigo and H. Coelho. Decisions with multiple simultaneous goals and uncertain causal effects. In Artificial Intelligence in Theory and Practice II, volume 276 of IFIP International Federation for Information Processing, pages 13-22. SpringerVerlag, 2008.

25. P. Trigo and H. Coelho. Simulating a multi-agent electricity market. In Proceedings of the 1st Brazilian Workshop on Social Simulation (BWSS-08 / SBIA-08), Bahia, Brazil, Oct 26-30 2008.

26. P. Trigo and H. Coelho. Agent inferencing meets the semantic web. In Progress in Artificial Intelligence, EPIA-09, volume 5816 of Lecture Notes in Artificial Intelligence (LNAI), pages 497-507. Springer-Verlag, 2009.

27. P. Trigo and H. Coelho. Decision making with hybrid models: the case of collective and individual motivations. International Journal of Reasoning-based Intelligent Systems (IJRIS), 2(1):60-72, 2010.

28. P. Trigo, A. Jonsson, and H. Coelho. Coordination with collective and individual decisions. In J. S. Sichman, H. Coelho, and S. O. Rezende, editors, Advances in Artificial Intelligence, IBERAMIA/SBIA 2006, volume 4140 of Lecture Notes in Artificial Intelligence - LNAI, pages 37-47. Springer-Verlag, Ribeirão Preto, Brasil, October 23-27 2006.

29. P. Trigo, P. Marques, and H. Coelho. (virtual) agents for running electricity markets. Simulation Modelling Practice and Theory Journal; Simulation-based Design and Evaluation of Multi-Agent Systems, to appear, 2010.

30. W. N. Venables and D. M. Smith. An Introduction to R. Network Theory Ltd publishing free software manuals, 2009.

31. M. Wooldridge. Reasoning About Rational Agents, chapter Implementing Rational Agents. The MIT Press, 2000. 